\title{
Research on Plane Advertisement Design based on Color Semantic and Perceptual Graphic Elements
}

\author{
Zhou Liping \\ Jiujiang university, Jiujiang 332005,China \\ Xuexi123@163.com
}

Keywords: Color semantics; Graphic elements; Plane ads; Global threshold; Image segmentation.

\begin{abstract}
Graphic element is one of the most important visual languages in advertising design, and it is the main carrier of emotion and content in emotional advertising. Color is one of the three important elements in the design of print ads, which plays an important role in print advertising. In order to study the effect of color semantic and emotional graphics in print advertising, this paper proposes a new method of image feature extraction, which uses global threshold, so as to realize the analysis of color semantic and graphic elements. Finally, this method is used to analyze the case of print advertising. From the result it can be seen that the reasonable application of the color semantic and perceptual graphic elements can improve the visual effect of the plane advertising and improve the advertising effectiveness.
\end{abstract}

\section{Introduction}

In the first 20 seconds of focused on the object, the first concern is the color of things, with the extension of time, graphic factors gradually enter into the visual perception, and began to lead to emotional experience [1-3]. Different colors can generate different information symbols, which cause people's emotional resonance, resulting in a more intense psychological change, to achieve different advertising effectiveness. Graphic element is one of the most important visual languages in advertisement design, and it is the main transmission carrier of advertising emotion and content $[4,5]$. The designer can use graphic elements to put perceptual graphics into the emotions and feelings, which can convey thought and emotion and produce good advertising effect, improving the advertising visual effect and spread ability. So it has important significance for the study of color semantics and graphical elements in the plane advertisement design.

\section{Advertising Features based on Color Semantic and Perceptual Elements}

Symbolic value of color. In daily life, people can also feel the psychological impact of color, through the subtle way, which can affect people's emotions and will. When the color produces emotional reaction through the visual, it has the spirit of advertising value.

The associative advertising value of color semantics. The color association refers to a variety of human sensory organs, for a specific color, triggering the feeling of experience. Through the association, it can trigger the related emotions and feelings, resulting in a sense of contact with a certain thing, so that the advertising value of the color is generated.

Visual effect of perceptual image element. In advertising design, graphic language is a very important way of expression, so the designers can improve their own cultural heritage through a wide range of reading and accumulate material through the experience of life, so as to display the emotional image elements creativity.

Excellent communication skills of perceptual image elements. In graphic advertising design, perceptual graphics language can effectively convey the core idea of advertising works, the performance of the technique is more intuitive. It can use vivid images to display the characteristics of things, so as to enhance the persuasiveness and infectivity of advertising. 


\section{Print Advertisement Image Analysis Method based on Global Threshold}

In order to analyze the color semantic and perceptual graphic elements in planar ad images, this paper proposes a global threshold analysis method, and does local binarization on the image, so the feature extraction effect of the image is enhanced. The global threshold compared with each pixel of the image [6]. If the value is more than the threshold, it is the front view; if it is less than the threshold, it is the background color. Assuming an image $N \times M, f(i, j)$ is the gray value of $(i, j)$, its gray level is $\mu$, and the value is $[0, m-1], p(k)$ is frequency of gray value $k$.

$$
p(k)=\frac{1}{M N} \sum_{f(i, j)=k} 1 .
$$

Assuming that the threshold of the gray value is $t$, the foreground and background of the local image is $\{f(i, j) \leq t\}$ and $\{f(i, j)>t\}$, so the target proportional of the local image is:

$$
\omega_{0}(t)=\sum_{0 \leq i \leq t} p(i)
$$

Target part number is:

$$
N_{0}(t)=M N \sum_{0 \leq i \leq t} p(i) \text {. }
$$

The foreground proportion is:

$$
\omega_{1}(t)=\sum_{t<i \leq m-1} p(i)
$$

The background part number is:

$$
N_{1}(t)=M N \sum_{t<i \leq m-1} P(i) .
$$

The target mean value is:

$$
\mu_{0}(t)=\sum_{0 \leq i \leq t} i p(i) / \omega_{0}(t)
$$

The background mean value is:

$$
\mu_{1}(t)=\sum_{t<i \leq m-1} i p(i) / \omega_{1}(t) .
$$

Then the total means value can be expressed as:

$$
\mu=\omega_{0}(t) \mu_{0}(t)+\omega_{1}(t) \mu_{1}(t) .
$$

In the analysis of the color semantic and perceptual graphics elements, the optimal threshold of image can be expressed as $g$, and the expression is:

$$
g=\operatorname{Arg} \underset{0 \leq t \leq m-1}{\operatorname{Max}}\left[\omega_{0}(t)\left(\mu_{0}(t)-\mu\right)^{2}+\omega_{1}(t)\left(\mu_{1}(t)-\mu\right)^{2}\right]
$$

Among then, $\mu_{0}(t)$ represents the target value, the probability is $\omega_{1}(t)$, the background value is $\mu_{1}(t)$, the probability is $\omega_{0}(t)$, the total mean is $\mu$. In order to make the feature extraction of the color semantics and the image not affecting the image, the segmentation process target should be far from the center of the image, so the value of $\left(\mu_{0}(t)-\mu\right)^{2}$ and $\left|\mu_{0}(t)-\mu\right|$ should be bigger, the background is also far from the image center, $\left(\mu_{1}(t)-\mu\right)^{2}$ and $\left|\mu_{1}(t)-\mu\right|$ are also bigger. In order to meet the requirements, the two are weighted and the weight is the biggest, so it is: 
$g=\operatorname{Arg} \underset{0 \leq t \leq \mathrm{m}-1}{\operatorname{Max}}\left[\omega_{0}(t)\left(\mu_{0}(t)-\mu\right)^{2}+\omega_{1}(t)\left(\mu_{1}(t)-\mu\right)^{2}\right]$

The product is the biggest, so

$g=\operatorname{Arg} \underset{0 \leq t \leq \mathrm{m}-1}{\operatorname{Max}}\left[\left(\mu_{0}(t)-\mu\right)^{2}\left(\mu_{1}(t)-\mu\right)^{2}\right]$.

It uses the weight threshold to extract image feature element, then carries on the analysis on the whole image of color and graphics, so it can get the role of two elements in the plane advertisement, the process is as shown in Figure 1.

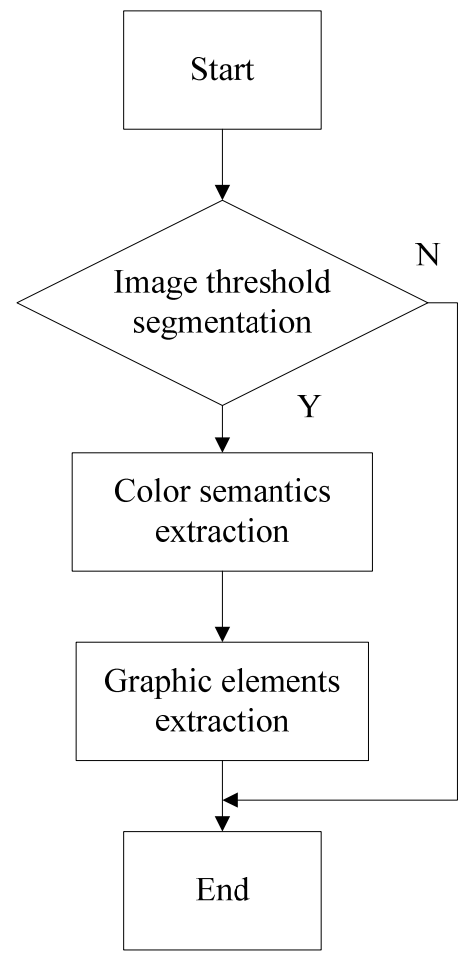

Fig.1 The design and analysis process of the planar advertisement element

In order to better design the print ads, we need to analyze the color and perceptual image elements, so that the two elements are better used in advertising design [7, 8]. The process is to set the threshold of the image, and then extract the color and image elements, analyze print advertising image through color semantics and graphic elements, it will be better applied to advertising design, which can improve the visual effect of advertising and the effectiveness of advertising.

\section{Case Analysis of Plane Advertisement}

In order to study the effect of color semantics and emotional graphics elements in ad design, in the guidance of the clustering feature, this paper studies them plane advertisement with different color semantics and color elements, and verifies the importance.

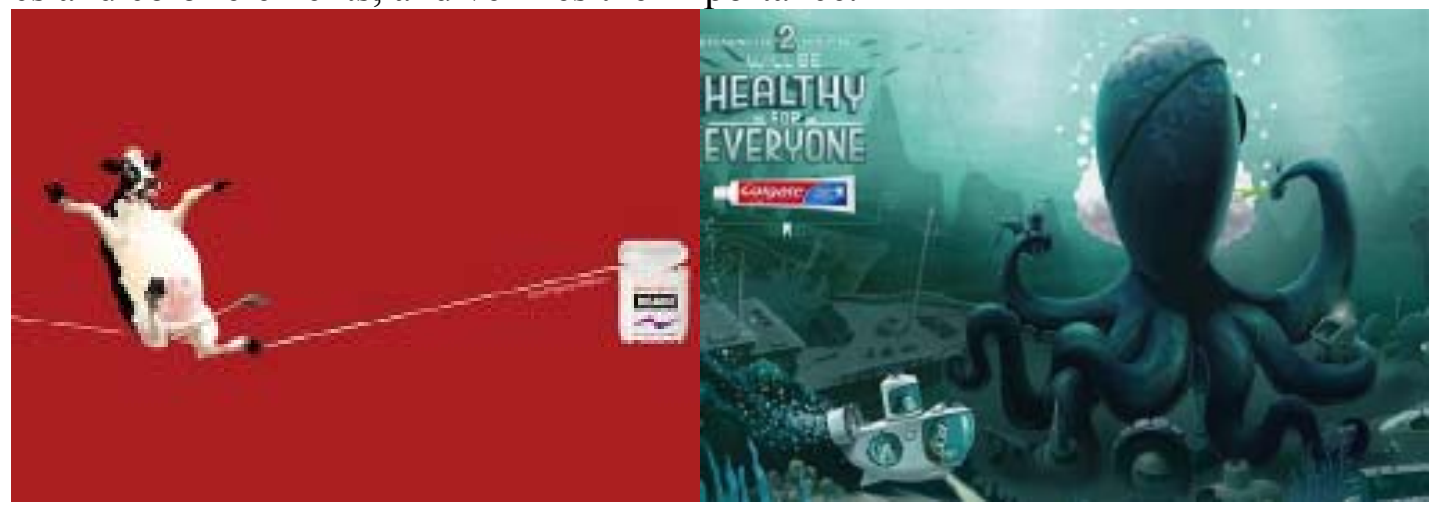

Fig.2 Cold warm comparison of color semantic advertising 
As shown in Figure 2, $\mathrm{t}$ is cold warm contrast of color semantics. Advertising is closely related with daily life, in daily life, when you see the ocean, ice and other natural things, it lets us feel the cold. These things are in blue and green as the main color tone [9]. The sun and the flame will give people warm feeling, and the corresponding colors are red and orange .In Figure 2 it can be seen the warm and cool colors in the advertising, and it gives the different visual impact.

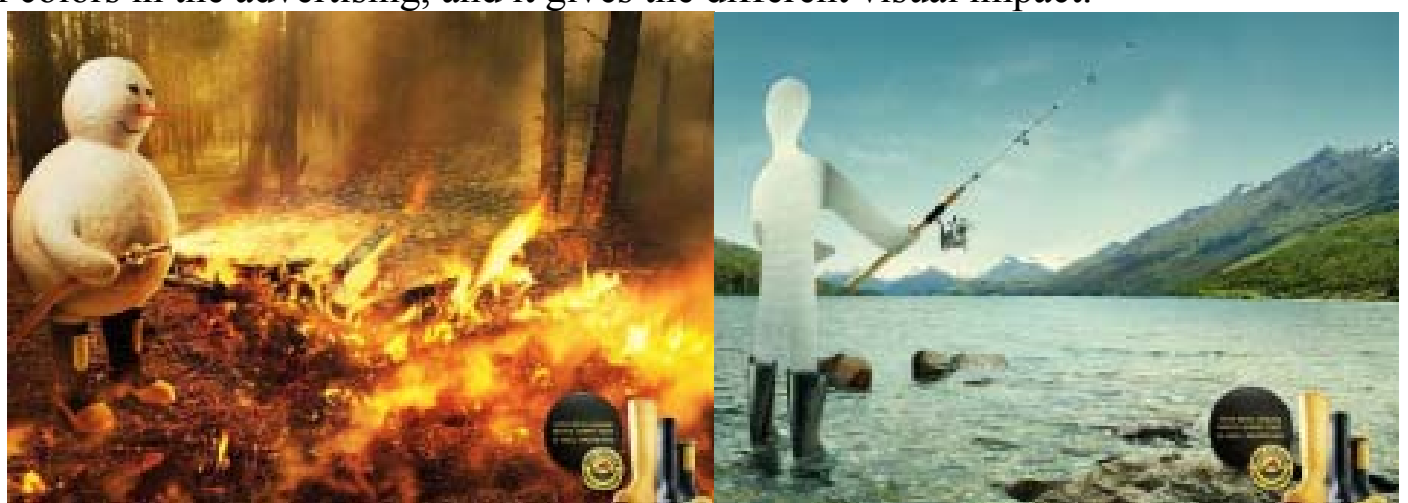

Fig.3 Exciting color and silence color in color semantic advertising

Figure 3 shows the impact of exciting colors and silence effect in the color semantic advertising, the embodiment is its main color lightness [10]. High brightness will have a sense of excitement, and low brightness of the color will generally produce a feeling of calm. General red, orange, yellow and other colors will give people with exciting feeling; green, blue, violet blue color will give people calm feeling.

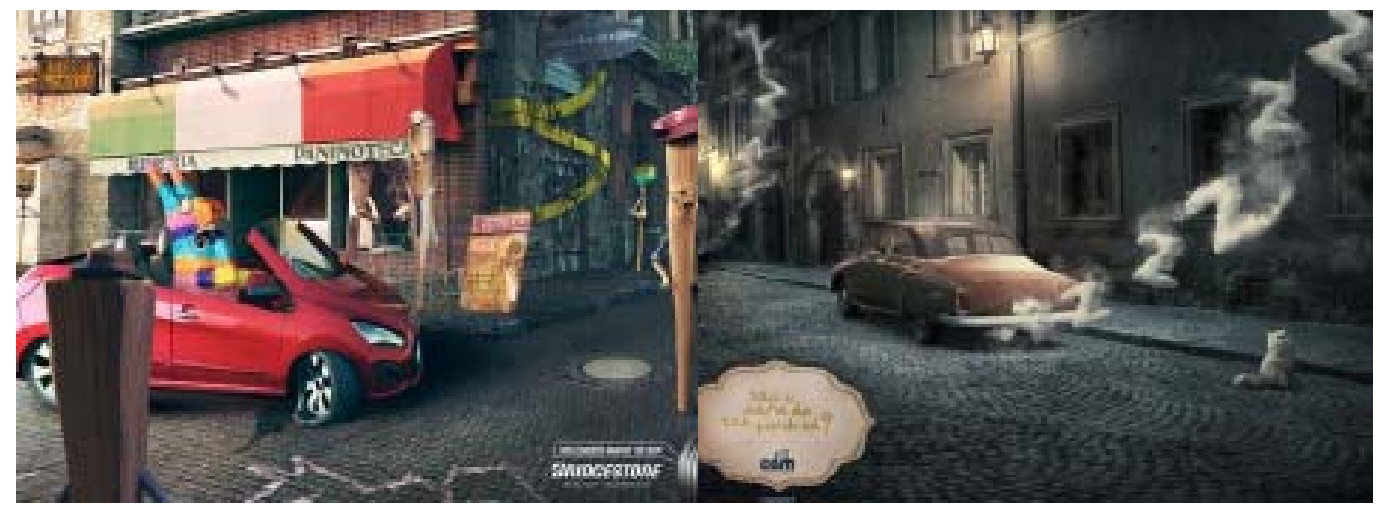

Fig.4 Contrast of positive and negative colors in color semantic advertising

Figure 4 shows the contrast effect of positive and negative colors in color semantic advertising. The embodiment is its main color lightness and purity, high purity and brightness will produce positive feelings, low purity and brightness will exert a positive negative feeling [11]. Color can have a positive sense, and relatively free of color will have a negative sense.

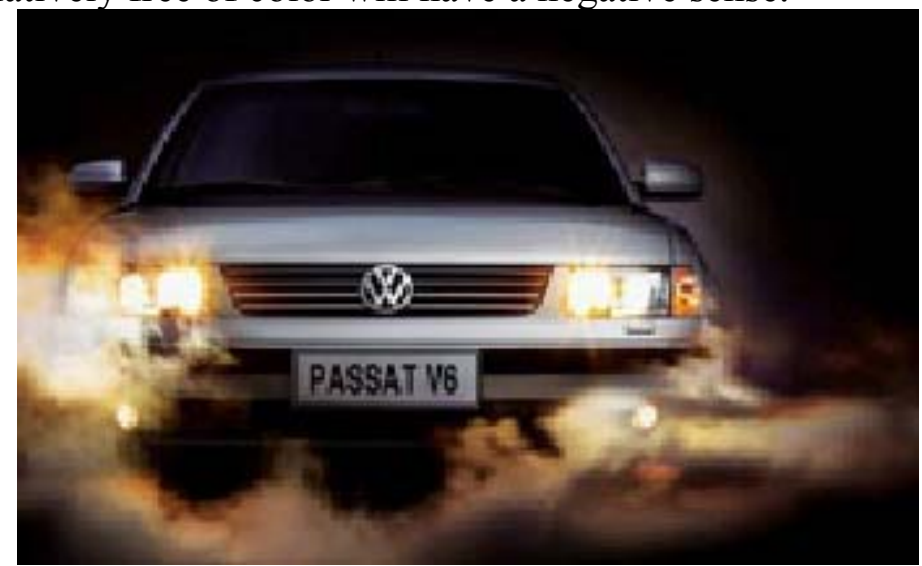

Fig.5 Figurative perceptual graphics elements

Figure 5 shows the effect of emotional graphics elements in advertising design. Figurative graphic elements are created graphics by people through processing, induction and landscaping [12]. In the communication of advertising information it will achieve better results and stimulate people's desire 
and interest, with strong visual impact and shock. In the modern graphic design it occupies an important position.

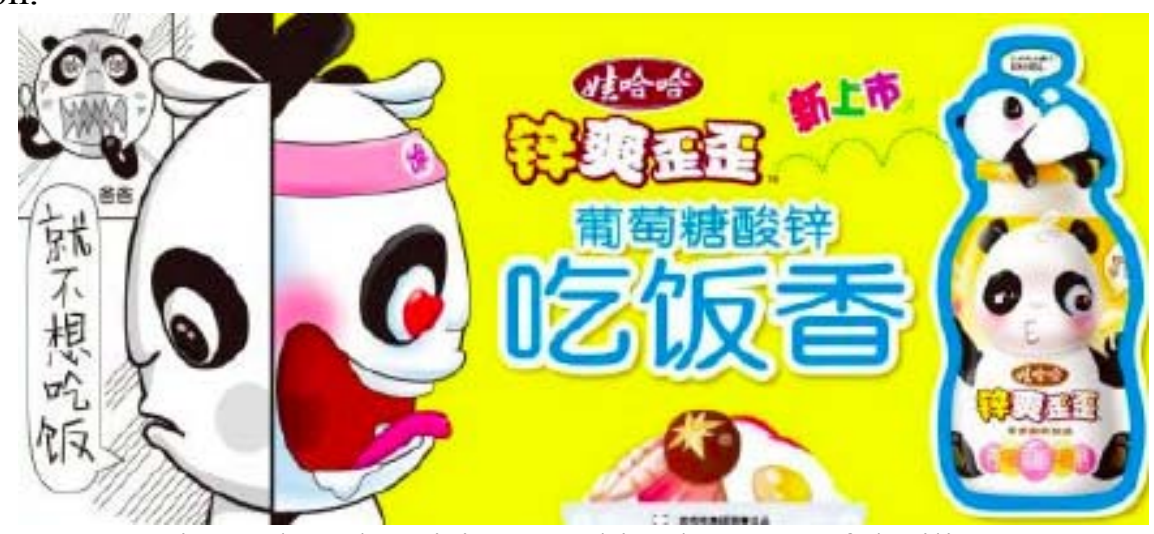

Fig.6 The advertising graphic elements of decilious

In children's advertising design, graphic elements are very important. The emotional needs of children can be more easily accepted, for example, when they see the food or the players' ads, they will have a strong desire to consume, so that parents are put forward spending intentions and requirements, which gets a good advertising effectiveness.

\section{Summary}

Based on the principle of image global threshold segmentation, combining with the color semantics and graphic elements, this paper analyzes the design of planar advertisement. According to the global threshold, it extracts the color semantics and image elements, and analyzes the plane advertisement. The results show that the color semantics and graphic elements can be applied to the advertising design, and it can effectively improve the impact and shock effect of plane advertisement, which provides a valuable reference for the data of plane advertisement.

\section{References}

[1] H. Liu, M.S. Zhong, L. Liu. The quantitative calculation method of semantic relations among words. Journal of Chinese information, 2014, 23 (2): 115-122.

[2] J.P. Sun, Y.C. Li. Research on image boundary problem based on wavelet transforms. Journal of Xi'an University of science and technology, 2013, 3 (3): 81-84.

[3] D.L. Wang, X.Y. Tu, J.T. Zi. Collocation extraction approach with fusion of multi strategy. Journal of Tsinghua University, 2013, 48 (4): 608-612.

[4] D.L. Wang, D.Z. Zhang, Z.J. Tong. Collocation extraction method based on relative conditional entropy. Journal of Beijing University of Posts and Telecommunications, 2013, 2(6): 40-45.

[5] K.L. Sun, W.C. Zhou, Q.Z. Wu. Engineering implementation of high speed real time optical fiber image transmission system. Optics and precision engineering, 2014, 19 (9): 2236-2242.

[6] X.B. Wang, Y. Zhu, B.X. Zheng. Research and implementation of CompactPCI Express communication interface module design based on FPGA. Electronic technology, 2013, 12(1): 119-123.

[7] K.L. Sun, W.C. Zhou, Q.Z. Wu. The high speed serial optical fiber image transmission detection platform based on FPGA. Semiconductor optoelectronics, 2013, 33(2): 283-287.

[8] M.G. Yang, Z.J. Zhai, H.M. Jiang. The multi parameter chaotic time series LS-SVR weighted prediction based on new information. Journal of Northwestern Polytechnic University, 2013, 3(1): 1125-1130. 
[9] X.L. Song, Q. Wang. Digital image parallel preprocessing based on GPGPU. Computer measurement and control, 2013, 17 (6): 1169-1171.

[10] Z.Y. Liu, M.S. Sun. The Chinese word co-occurrence network of small world effect and scale-free characteristics. Chinese information journal, 2013,21(6): 52-58.

[11] H. Geng, P. Zhao, Q.S. Cai. K-means clustering algorithm based on weighted complex network features. Computer technology and development, 2013,17(9): 35-37.

[12] J. Guo, Q.K. Chen. fast image compression based on CUDA. Computer engineering and design, 2014,31(14): 3302-3304. 